DOI: 10.17516/1997-1397-2021-14-3-313-325

УДК 517.9

\title{
Uniqueness and Stability Results for Caputo Fractional Volterra-Fredholm Integro-Differential Equations
}

\author{
Ahmed A. Hamoud* \\ Department of Mathematics \\ Taiz University \\ Taiz, Yemen
}

Received 10.08.2020, received in revised form 10.01.2021, accepted 20.03.2021

\begin{abstract}
In this paper, we established some new results concerning the uniqueness and Ulam's stability results of the solutions of iterative nonlinear Volterra-Fredholm integro-differential equations subject to the boundary conditions. The fractional derivatives are considered in the Caputo sense. These new results are obtained by applying the Gronwall-Bellman's inequality and the Banach contraction fixed point theorem. An illustrative example is included to demonstrate the efficiency and reliability of our results.
\end{abstract}

Keywords: Volterra-Fredholm integro-differential equation, Caputo sense, Gronwall-Bellman's inequality, Banach contraction fixed point theorem.

Citation: A.A.Hamoud, Uniqueness and Stability Results for Caputo Fractional Volterra-Fredholm Integro-Differential Equations, J. Sib. Fed. Univ. Math. Phys., 2021, 14(3), 313-325.

DOI: $10.17516 / 1997-1397-2021-14-3-313-325$.

\section{Introduction}

In recent years, there has been a growing interest in the linear and nonlinear integrodifferential equations which are a combination of differential and integral equations [3,16,18,21]. The nonlinear integro-differential equations play an important role in many branches of nonlinear functional analysis and their applications in the theory of engineering, mechanics, physics, electrostatics, biology, chemistry and economics [13] and signal processing [25].

The challenging work is to find the solution while dealing with Volterra-Fredholm fractional integro-differential equations. Therefore, many researchers have tried their best to use different techniques to find the analytical and numerical solutions of these problems $[1,2,4,6-8,10,14,22$, $23,29]$.

The study of iterative differential and integro-differential equations is linked to the wide applications of calculus in mathematical sciences. These equations are vital in the study of infection models. Many papers have dealt with the existence, uniqueness and other properties of solutions of special forms of the iterative differential equations and integro-differential equations $[12,15,16,19,20]$.

Recently, Cheng et al. (2009), in [5,20] investigated analytic and exact solutions of an iterative functional differential equation

$$
\begin{aligned}
& u^{\prime}(x)=f(x, u(h(x)+g(u(x)))), \\
& u\left(x_{0}\right)=x_{0} .
\end{aligned}
$$

*drahmedselwi985@hotmail.com https://orcid.org/0000-0002-8877-7337

(c) Siberian Federal University. All rights reserved 
Lauran (2011) [19], investigated the existence and uniqueness results for first order differential and iterative differential equations with deviating argument of the type

$$
\begin{aligned}
& u^{\prime}(t)=f(t, u(t), u(u(t)), u(\lambda u(t))), \\
& u\left(t_{0}\right)=x_{0} .
\end{aligned}
$$

In [15], Ibrahim (2013) investigated the existence and uniqueness of solution for iterative differential equations of the type

$$
\begin{aligned}
& D^{\alpha} u(t)=f(t, u(u(t))), \\
& u(0)=u_{0} .
\end{aligned}
$$

Kendre et al. (2015), [16] investigated the existence of solution for iterative integro-differential equations of the type

$$
\begin{aligned}
& u^{\prime}(t)=f\left(t, u(u(t)), \int_{t_{0}}^{t} k(t, s) u(u(s)) d s\right), \\
& u\left(t_{0}\right)=x_{0} .
\end{aligned}
$$

Unhale and Kendre (2019), in [28] established the existence and uniqueness of solution for iterative integro-differential equations of the type

$$
\begin{aligned}
& D^{\alpha} u(t)=f(t)+\int_{0}^{t} h(t, s) u(\lambda u(s)) d s, \\
& u(0)=u_{0} .
\end{aligned}
$$

Motivated by these problems, in this paper, we discuss new uniqueness and stability results for nonlinear fractional Volterra-Fredholm integro-differential equation with deviating argument of the type

$$
D^{\alpha} u(x)=f(x)+\int_{0}^{x} h(x, s) u(u(s)) d s+\int_{0}^{T} k(x, s) u(u(s)) d s, \quad x, s \in J:=[0, T],
$$

with the boundary condition

$$
a u(0)+b u(T)=c, \quad a, b, c \in \mathbb{R}, a+b \neq 0,
$$

where $D^{\alpha}(),. 0<\alpha<1$, is the Caputo fractional derivative, $f(t), h(x, s)$ and $k(x, s)$ are given continuous functions, $u(x)$ is the unknown function to be determined.

The main objective of the present paper is to study the new uniqueness and stability results for iterative nonlinear fractional Volterra-Fredholm integro-differential equation with deviating argument.

The rest of the paper is organized as follows: In Section 1, some essential notations, definitions and Lemmas related to fractional calculus are recalled. In Section 2, the new uniqueness and stability results of the solution for nonlinear fractional Volterra-Fredholm integro-differential equation have been proved. In Section 3, we investigate the Ulam-Hyers stability and generalized Ulam-Hyers stability for the problem (1)-(2). In Section 4, focuses on an example to illustrate the theory. Finally, we will give a report on our paper and a brief conclusion.

\section{Preliminaries}

The mathematical definitions of fractional derivative and fractional integration are the subject of several different approaches. The most frequently used definitions of the fractional calculus involves the Riemann-Liouville fractional derivative, Caputo derivative, etc. The following observations are taken from [7, 9-11, 17, 18, 24, 26, 29]. 
Definition 1.1 ([16]). The Riemann-Liouville fractional integral of order $\alpha>0$ of a function $f$ is defined as

$$
\begin{aligned}
& J^{\alpha} f(x)=\frac{1}{\Gamma(\alpha)} \int_{0}^{x}(x-t)^{\alpha-1} f(t) d t, \quad x>0, \quad \alpha \in \mathbb{R}^{+}, \\
& J^{0} f(x)=f(x)
\end{aligned}
$$

where $\mathbb{R}^{+}$is the set of positive real numbers.

Definition 1.2 ([16]). The Riemann-Liouville derivative of order $\alpha$ with the lower limit zero for a function $f:[0,1) \longrightarrow \mathbb{R}$ can be written as

$$
{ }^{L} D^{\alpha} f(x)=\frac{1}{\Gamma(1-\alpha)} \frac{d}{d x} \int_{0}^{x} \frac{f(t)}{(x-t)^{\alpha}} d t, \quad x>0, \quad 0<\alpha<1 .
$$

Definition 1.3 ([24]). The Caputo derivative of order $\alpha$ for a function $f:[0,1) \longrightarrow \mathbb{R}$ can be written as

$$
D^{\alpha} f(x)=\frac{1}{\Gamma(1-\alpha)} \int_{0}^{x} \frac{f^{\prime}(t)}{(x-t)^{\alpha}} d t, \quad x>0, \quad 0<\alpha<1 .
$$

Definition $1.4([26])$. The fractional derivative of $f(x)$ in the Caputo sense is defined by

$$
\begin{aligned}
{ }^{c} D^{\alpha} f(x) & =J^{n-\alpha} D^{n} f(x)= \\
& = \begin{cases}\frac{1}{\Gamma(n-\alpha)} \int_{0}^{x}(x-t)^{n-\alpha-1} \frac{d^{n} f(t)}{d t^{n}} d t, & n-1<\alpha<n, \\
\frac{d^{n} f(x)}{d x^{n}}, & \alpha=n,\end{cases}
\end{aligned}
$$

where the parameter $\alpha$ is the order of the derivative, in general it is real or even complex.

Definition 1.5 ([26]). The Riemann-Liouville fractional derivative of order $\alpha>0$ is normally defined as

$$
D^{\alpha} f(x)=D^{m} J^{m-\alpha} f(x), \quad m-1<\alpha \leqslant m .
$$

Lemma 1.1 ([24], Gronwall-Bellman's Inequality). Let $u(x)$ and $f(x)$ be nonnegative continuous functions defined on $J=[\alpha, \alpha+h]$ and $c$ be a nonnegative constant. If

$$
u(x) \leqslant c+\int_{\alpha}^{x} f(s) u(s) d s, \quad x \in J
$$

then

$$
u(x) \leqslant c \exp \left(\int_{\alpha}^{x} f(s) d s\right), \quad x \in J
$$

Theorem 1.1 ([26], Banach contraction principle). Let $(X, d)$ be a complete metric space, then each contraction mapping $\mathcal{T}: X \longrightarrow X$ has a unique fixed point $x$ of $\mathcal{T}$ in $X$ i.e. $\mathcal{T} x=x$.

\section{Main results}

In this section, we shall give an existence and uniqueness results of Eq. (1), with the boundary condition (2). Let $B=C(J, J)$ be the Banach space equipped with the norm 
$\|u\|=\max _{x \in[0, T]}|u(x)|$. For convenience, we are listing the following hypotheses used in our further discussion: (A1) There exist two constants $\beta_{h}$ and $\beta_{k}$ such that

$$
\begin{aligned}
& \beta_{h}=\sup \{|h(t, s)|: 0 \leqslant s \leqslant t \leqslant T\} . \\
& \beta_{k}=\sup \{|k(t, s)|: 0 \leqslant s \leqslant t \leqslant T\} .
\end{aligned}
$$

(A2) There exists a constant $M>0$ such that

$$
\left|u\left(t_{1}\right)-u\left(t_{2}\right)\right| \leqslant M\left|t_{1}-t_{2}\right|^{\alpha}, \text { for } u \in B, t_{1}, t_{2} \in J, t_{1} \leqslant t_{2} .
$$

(A3) There exists a constant $L>0$ such that $L=\sup \{|f(t)|: 0 \leqslant t \leqslant T\}$.

(A4) Let $\rho:=\frac{T^{\alpha}\left(L+T^{3}\left(\beta_{h}+\beta_{k}\right)\right)}{\Gamma(\alpha+1)}\left[1+\frac{|b|}{|a+b|}\right]+\frac{|c|}{|a+b|} \leqslant T \leqslant M$.

Lemma 2.1. If a function $u \in C[0, T]$ satisfies (1)-(2) in the closed interval $[0, T]$, then the problems (1)-(2) are equivalent to the problem of finding a continuous solution of the integral equation

$$
\begin{aligned}
u(x)= & \int_{0}^{x} \frac{(x-t)^{\alpha-1}}{\Gamma(\alpha)}\left(f(t)+\int_{0}^{t} h(t, s) u(u(s)) d s+\int_{0}^{T} k(t, s) u(u(s)) d s\right) d t- \\
& -\frac{1}{a+b}\left[\int_{0}^{T} \frac{b(T-t)^{\alpha-1}}{\Gamma(\alpha)}\left(f(t)+\int_{0}^{t} h(t, s) u(u(s)) d s+\int_{0}^{T} k(t, s) u(u(s)) d s\right) d t-c\right] .
\end{aligned}
$$

Theorem 2.1. Suppose that the hypotheses (A1)-(A4) are satisfied and

$$
\left[\frac{T^{\alpha+1}\left(\beta_{h}+\beta_{k}\right)(M+1)}{\Gamma(\alpha+1)}\left(1+\frac{|b|}{|a+b|}\right)\right]<1 .
$$

Then there is a unique solution to the problems (1)-(2).

Proof. Let $B(\rho)=\left\{u \in B: 0 \leqslant u \leqslant \rho,\left|u\left(t_{1}\right)-u\left(t_{2}\right)\right| \leqslant M\left|t_{1}-t_{2}\right|^{\alpha}\right\}$.

To apply Banach contraction principle, we define an operator $\Psi: B(\rho) \longrightarrow B(\rho)$ by

$$
\begin{aligned}
(\Psi u)(x) & =\int_{0}^{x} \frac{(x-t)^{\alpha-1}}{\Gamma(\alpha)}\left(f(t)+\int_{0}^{t} h(t, s) u(u(s)) d s+\int_{0}^{T} k(t, s) u(u(s)) d s\right) d t- \\
& -\frac{1}{a+b}\left[\int_{0}^{T} \frac{b(T-t)^{\alpha-1}}{\Gamma(\alpha)}\left(f(t)+\int_{0}^{t} h(t, s) u(u(s)) d s+\int_{0}^{T} k(t, s) u(u(s)) d s\right) d t-c\right] .
\end{aligned}
$$


So, we have

$$
\begin{aligned}
0 \leqslant|\Psi u| & =\mid \int_{0}^{x} \frac{(x-t)^{\alpha-1}}{\Gamma(\alpha)}\left(f(t)+\int_{0}^{t} h(t, s) u(u(s)) d s+\int_{0}^{T} k(t, s) u(u(s)) d s\right) d t- \\
& -\frac{1}{a+b}\left[\int_{0}^{T} \frac{b(T-t)^{\alpha-1}}{\Gamma(\alpha)}\left(f(t)+\int_{0}^{t} h(t, s) u(u(s)) d s+\int_{0}^{T} k(t, s) u(u(s)) d s\right) d t-c\right] \mid \leqslant \\
& \leqslant \int_{0}^{x} \frac{(x-t)^{\alpha-1}}{\Gamma(\alpha)}\left(|f(t)|+\int_{0}^{t}|h(t, s)||u(u(s))| d s+\int_{0}^{T}|k(t, s)||u(u(s))| d s\right) d t+ \\
& +\frac{1}{|a+b|} \int_{0}^{T} \frac{|b|(T-t)^{\alpha-1}}{\Gamma(\alpha)}\left(|f(t)|+\int_{0}^{t}|h(t, s)||u(u(s))| d s+\int_{0}^{T}|k(t, s)||u(u(s))| d s\right) d t+ \\
& +\frac{|c|}{|a+b|} \leqslant \\
& \leqslant \int_{0}^{x} \frac{(x-t)^{\alpha-1}}{\Gamma(\alpha)}\left(L+\left(\beta_{h}+\beta_{k}\right) T^{3}\right) d s+\frac{1}{|a+b|} \int_{0}^{T} \frac{|b|(T-t)^{\alpha-1}}{\Gamma(\alpha)}\left(L+\left(\beta_{h}+\beta_{k}\right) T^{3}\right) d t+ \\
& +\frac{|c|}{|a+b|} \leqslant \\
\leqslant & \frac{T^{\alpha}\left(L+T^{3}\left(\beta_{h}+\beta_{k}\right)\right)}{\Gamma(\alpha+1)}\left[1+\frac{|b|}{|a+b|}\right]+\frac{|c|}{|a+b|}= \\
& =\rho .
\end{aligned}
$$

Also, for each $0 \leqslant x_{1} \leqslant x_{2} \leqslant T$, we have

$$
\begin{aligned}
& \left|\Psi u\left(x_{2}\right)-\Psi u\left(x_{1}\right)\right| \leqslant \\
& \quad \leqslant\left|\int_{0}^{x_{1}} \frac{\left(x_{2}-t\right)^{\alpha-1}-\left(x_{1}-t\right)^{\alpha-1}}{\Gamma(\alpha)}\left(f(t)+\int_{0}^{t} h(t, s) u(u(s)) d s+\int_{0}^{T} k(t, s) u(u(s)) d s\right) d t\right|+ \\
& \quad+\left|\int_{x_{1}}^{x_{2}} \frac{\left(x_{2}-t\right)^{\alpha-1}}{\Gamma(\alpha)}\left(f(t)+\int_{0}^{t} h(t, s) u(u(s)) d s+\int_{0}^{T} k(t, s) u(u(s)) d s\right) d t\right| \leqslant \\
& \quad \leqslant \frac{1}{\Gamma(\alpha)} \int_{0}^{x_{1}}\left[\left(x_{1}-t\right)^{\alpha-1}-\left(x_{2}-t\right)^{\alpha-1}\right]\left(|f(t)|+\int_{0}^{t}|h(t, s)||u(u(s))| d s+\right. \\
& \left.\quad+\int_{0}^{T}|k(t, s)||u(u(s))| d s\right) d t+\frac{1}{\Gamma(\alpha)} \int_{x_{1}}^{x_{2}}\left(x_{2}-t\right)^{\alpha-1}\left(|f(t)|+\int_{0}^{t}|h(t, s)||u(u(s))| d s+\right. \\
& \left.\quad+\int_{0}^{T}|k(t, s)||u(u(s))| d s\right) d t .
\end{aligned}
$$

Hence,

$$
\begin{aligned}
\left|\Psi u\left(x_{2}\right)-\Psi u\left(x_{1}\right)\right| & \leqslant \frac{1}{\Gamma(\alpha)} \int_{0}^{x_{1}}\left[\left(x_{1}-t\right)^{\alpha-1}-\left(x_{2}-t\right)^{\alpha-1}\right]\left[L+T^{3}\left(\beta_{h}+\beta_{k}\right)\right] d t+ \\
& +\frac{1}{\Gamma(\alpha)} \int_{x_{1}}^{x_{2}}\left(x_{2}-t\right)^{\alpha-1}\left[L+T^{3}\left(\beta_{h}+\beta_{k}\right)\right] d t \leqslant \\
& \leqslant \frac{\left[L+T^{3}\left(\beta_{h}+\beta_{k}\right)\right]}{\Gamma(\alpha+1)}\left[x_{1}^{\alpha}-x_{2}^{\alpha}+2\left(x_{2}-x_{1}\right)^{\alpha}\right] \leqslant \\
& \leqslant \frac{2\left[L+T^{3}\left(\beta_{h}+\beta_{k}\right)\right]}{\Gamma(\alpha+1)}\left|x_{2}-x_{1}\right|^{\alpha}
\end{aligned}
$$


This shows that $\Psi$ maps from $B(\rho) \longrightarrow B(\rho)$. Now, for all $u, v \in B(\rho)$, we have

$$
\begin{aligned}
& |\Psi u(x)-\Psi v(x)| \leqslant \\
\leqslant & \frac{1}{\Gamma(\alpha)} \int_{0}^{x}(x-t)^{\alpha-1}\left(\int_{0}^{t}|h(t, s)||u(u(s))-v(v(s))| d s+\int_{0}^{T}|k(t, s)||u(u(s))-v(v(s))| d s\right) d t+ \\
& +\frac{|b|}{|a+b| \Gamma(\alpha)} \int_{0}^{T}(T-t)^{\alpha-1}\left(\int_{0}^{t}|h(t, s)||u(u(s))-v(v(s))| d s+\right. \\
& \left.+\int_{0}^{T}|k(t, s)||u(u(s))-v(v(s))| d s\right) d t \leqslant \\
\leqslant & \frac{\left(\beta_{h}+\beta_{k}\right)}{\Gamma(\alpha)} \int_{0}^{x}(x-t)^{\alpha-1}\left(\int_{0}^{t}|u(u(s))-u(v(s))|+|u(v(s))-v(v(s))| d s+\right. \\
& \left.+\int_{0}^{T}|u(u(s))-u(v(s))|+|u(v(s))-v(v(s))| d s\right) d t+ \\
& +\frac{|b|\left(\beta_{h}+\beta_{k}\right)}{|a+b| \Gamma(\alpha)} \int_{0}^{T}(T-t)^{\alpha-1}\left(\int_{0}^{t}|u(u(s))-u(v(s))|+|u(v(s))-v(v(s))| d s+\right. \\
& \left.+\int_{0}^{T}|u(u(s))-u(v(s))|+|u(v(s))-v(v(s))| d s\right) d t \leqslant \\
\leqslant & \frac{\left(\beta_{h}+\beta_{k}\right)}{\Gamma(\alpha)} \int_{0}^{x}(x-t)^{\alpha-1}\left(\int_{0}^{t}(M|u(s)-v(s)|+|u(s)-v(s)|) d s\right) d t+ \\
& +\frac{|b|\left(\beta_{h}+\beta_{k}\right)}{|a+b| \Gamma(\alpha)} \int_{0}^{T}(T-t)^{\alpha-1}\left(\int_{0}^{t}(M|u(s)-v(s)|+|u(s)-v(s)|) d s\right) d t \leqslant \\
\leqslant & \frac{\left(\beta_{h}+\beta_{k}\right)}{\Gamma(\alpha)} \int_{0}^{x}(x-t)^{\alpha-1}\left(\int_{0}^{t}((M+1)|u(s)-v(s)|) d s\right) d t+ \\
& +\frac{|b|\left(\beta_{h}+\beta_{k}\right)}{|a+b| \Gamma(\alpha)} \int_{0}^{T}(T-t)^{\alpha-1}\left(\int_{0}^{t}((M+1)|u(s)-v(s)|) d s\right) d t \leqslant \\
\leqslant & \frac{T\left(\beta_{h}+\beta_{k}\right)(M+1)}{\Gamma(\alpha)}\|u-v\| \int_{0}^{x}(x-t)^{\alpha-1} d t+\frac{|b| T\left(\beta_{h}+\beta_{k}\right)(M+1)}{\Gamma(\alpha+1)}\|u-v\| \int_{0}^{T}(T-t)^{\alpha-1} d t \leqslant \\
\leqslant & \left.\frac{T^{\alpha+1}\left(\beta_{h}+\beta_{k}\right)(M+1)}{\Gamma(\alpha+1)}\left(1+\frac{|b|}{|a+b|}\right)\right]\|u-v\| . \\
&
\end{aligned}
$$

Since

$$
\left[\frac{T^{\alpha+1}\left(\beta_{h}+\beta_{k}\right)(M+1)}{\Gamma(\alpha+1)}\left(1+\frac{|b|}{|a+b|}\right)\right]<1,
$$

by the Banach contraction principle, $\Psi$ has a unique fixed point. This means that the problems (1)-(2) has unique solution.

The above theorem shows that there exists a unique solution to the problems (1)-(2). However, it does not tell us how to find this solution. To find the solution of the problems (1)-(2), we will define the following sequence

$$
\begin{aligned}
& u_{n+1}(x)=\int_{0}^{x} \frac{(x-t)^{\alpha-1}}{\Gamma(\alpha)}\left(f(t)+\int_{0}^{t} h(t, s) u_{n}\left(u_{n}(s)\right) d s+\int_{0}^{T} k(t, s) u_{n}\left(u_{n}(s)\right) d s\right) d t- \\
& -\frac{1}{a+b}\left[\int_{0}^{T} \frac{b(T-t)^{\alpha-1}}{\Gamma(\alpha)}\left(f(t)+\int_{0}^{t} h(t, s) u_{n}\left(u_{n}(s)\right) d s+\int_{0}^{T} k(t, s) u_{n}\left(u_{n}(s)\right) d s\right) d t-c\right],
\end{aligned}
$$


where $n=0,1,2, \ldots$ and $u_{0}(x)$ is fixed functions of the class $C^{1}$ mapping $[0, T] \longrightarrow[0, T]$ such that $\left|u_{0}(x)\right| \leqslant T$. For this, we have the following theorem.

Theorem 2.2. If the assumptions of the Theorem 2.1 are satisfied then the sequences defined in (7) converges uniformly to the unique solution of the problems (1)-(2).

Proof. Let $U_{k}=\max _{x \in J}\left|u_{k}(x)-u_{k-1}(x)\right|$. Then

$$
\begin{aligned}
U_{1} & =\max _{x \in J}\left|u_{1}(x)-u_{0}(x)\right|= \\
& =\max _{x \in J} \mid \int_{0}^{x} \frac{(x-t)^{\alpha-1}}{\Gamma(\alpha)}\left(f(t)+\int_{0}^{t} h(t, s) u_{0}\left(u_{0}(s)\right) d s+\int_{0}^{T} k(t, s) u_{0}\left(u_{0}(s)\right) d s\right) d t- \\
- & \frac{1}{a+b}\left[\int_{0}^{T} \frac{b(T-t)^{\alpha-1}}{\Gamma(\alpha)}\left(f(t)+\int_{0}^{t} h(t, s) u_{0}\left(u_{0}(s)\right) d s+\int_{0}^{T} k(t, s) u_{0}\left(u_{0}(s)\right) d s\right) d t-c\right]-u_{0}(x) \mid \leqslant \\
& \leqslant \frac{T^{\alpha}\left(L+T^{3}\left(\beta_{h}+\beta_{k}\right)\right)}{\Gamma(\alpha+1)}\left(1+\frac{|b|}{|a+b|}\right)+\frac{|c|}{|a+b|} \leqslant
\end{aligned}
$$

$\leqslant T$.

Since $u_{0}:[0, T] \longrightarrow[0, T]$, we have $U_{1} \leqslant T$.

$$
\begin{aligned}
U_{2} & =\max _{x \in J}\left|u_{2}(x)-u_{1}(x)\right|= \\
& =\max _{x \in J} \mid \int_{0}^{x} \frac{(x-t)^{\alpha-1}}{\Gamma(\alpha)}\left(f(t)+\int_{0}^{t} h(t, s) u_{1}\left(u_{1}(s)\right) d s+\int_{0}^{T} k(t, s) u_{1}\left(u_{1}(s)\right) d s\right) d t- \\
& -\frac{1}{a+b}\left[\int_{0}^{T} \frac{b(T-t)^{\alpha-1}}{\Gamma(\alpha)}\left(f(t)+\int_{0}^{t} h(t, s) u_{1}\left(u_{1}(s)\right) d s+\int_{0}^{T} k(t, s) u_{1}\left(u_{1}(s)\right) d s\right) d t-c\right]- \\
& -\left\{\int_{0}^{x} \frac{(x-t)^{\alpha-1}}{\Gamma(\alpha)}\left(f(t)+\int_{0}^{t} h(t, s) u_{0}\left(u_{0}(s)\right) d s+\int_{0}^{T} k(t, s) u_{0}\left(u_{0}(s)\right) d s\right) d t-\right. \\
& \left.-\frac{1}{a+b}\left[\int_{0}^{T} \frac{b(T-t)^{\alpha-1}}{\Gamma(\alpha)}\left(f(t)+\int_{0}^{t} h(t, s) u_{0}\left(u_{0}(s)\right) d s+\int_{0}^{T} k(t, s) u_{0}\left(u_{0}(s)\right) d s\right) d t-c\right] d t\right\} \mid \leqslant \\
& \leqslant \max _{x \in J}\left\{\int _ { 0 } ^ { x } \frac { ( x - t ) ^ { \alpha - 1 } } { \Gamma ( \alpha ) } \left(|f(t)|+\int_{0}^{t}|h(t, s)|\left|u_{1}\left(u_{1}(s)\right)-u_{0}\left(u_{0}(s)\right)\right| d s+\right.\right. \\
& \left.+\int_{0}^{T}|k(t, s)|\left|u_{1}\left(u_{1}(s)\right)-u_{0}\left(u_{0}(s)\right)\right| d s\right) d t-\frac{1}{|a+b|} \int_{0}^{T} \frac{|b|(T-t)^{\alpha-1}}{\Gamma(\alpha)} \times \\
& \left.\times\left(|f(t)|+\int_{0}^{t}|h(t, s)|\left|u_{1}\left(u_{1}(s)\right)-u_{0}\left(u_{0}(s)\right)\right| d s+\int_{0}^{T}|k(t, s)|\left|u_{1}\left(u_{1}(s)\right)-u_{0}\left(u_{0}(s)\right)\right| d s\right) d t\right\} \leqslant \\
& \leqslant T U_{1} \leqslant T^{2} .
\end{aligned}
$$

Assume that result is true for $n$ i.e. $U_{n} \leqslant T U_{n-1} \leqslant T^{n}$. Now, we show that result holds for $n+1$

$$
\begin{aligned}
U_{n+1} & =\max _{x \in J}\left|u_{n+1}(x)-u_{n}(x)\right|= \\
& =\max _{x \in J} \mid \int_{0}^{x} \frac{(x-t)^{\alpha-1}}{\Gamma(\alpha)}\left(f(t)+\int_{0}^{t} h(t, s) u_{1}\left(u_{n}(s)\right) d s+\int_{0}^{T} k(t, s) u_{n}\left(u_{n}(s)\right) d s\right) d t- \\
& -\frac{1}{a+b}\left[\int_{0}^{T} \frac{b(T-t)^{\alpha-1}}{\Gamma(\alpha)}\left(f(t)+\int_{0}^{t} h(t, s) u_{n}\left(u_{n}(s)\right) d s+\int_{0}^{T} k(t, s) u_{n}\left(u_{n}(s)\right) d s\right) d t-c\right]-
\end{aligned}
$$




$$
\begin{aligned}
& -\left\{\int_{0}^{x} \frac{(x-t)^{\alpha-1}}{\Gamma(\alpha)}\left(f(t)+\int_{0}^{t} h(t, s) u_{n-1}\left(u_{n-1}(s)\right) d s+\int_{0}^{T} k(t, s) u_{n-1}\left(u_{n-1}(s)\right) d s\right) d t-\right. \\
& -\frac{1}{a+b}\left[\int _ { 0 } ^ { T } \frac { b ( T - t ) ^ { \alpha - 1 } } { \Gamma ( \alpha ) } \left(f(t)+\int_{0}^{t} h(t, s) u_{n-1}\left(u_{n-1}(s)\right) d s+\right.\right. \\
& \left.\left.\left.+\int_{0}^{T} k(t, s) u_{n-1}\left(u_{n-1}(s)\right) d s\right) d t-c\right] d t\right\} \mid \leqslant \\
& \leqslant \max _{x \in J}\left\{\int _ { 0 } ^ { x } \frac { ( x - t ) ^ { \alpha - 1 } } { \Gamma ( \alpha ) } \left(|f(t)|+\int_{0}^{t}|h(t, s)|\left|u_{n}\left(u_{n}(s)\right)-u_{n-1}\left(u_{n-1}(s)\right)\right| d s+\right.\right. \\
& \left.+\int_{0}^{T}|k(t, s)|\left|u_{n}\left(u_{n}(s)\right)-u_{n-1}\left(u_{n-1}(s)\right)\right| d s\right) d t-\frac{1}{|a+b|} \int_{0}^{T} \frac{|b|(T-t)^{\alpha-1}}{\Gamma(\alpha)} \times \\
& \times\left(|f(t)|+\int_{0}^{t}|h(t, s)|\left|u_{n}\left(u_{n}(s)\right)-u_{n-1}\left(u_{n-1}(s)\right)\right| d s+\right. \\
& \left.\left.+\int_{0}^{T}|k(t, s)|\left|u_{n}\left(u_{n}(s)\right)-u_{n-1}\left(u_{n-1}(s)\right)\right| d s\right) d t\right\} \leqslant \\
& \leqslant T U_{n} \leqslant T^{n+1} .
\end{aligned}
$$

Thus by induction, we have $U_{k} \leqslant T^{k}$. Since

$$
\frac{T^{\alpha}\left(L+T^{3}\left(\beta_{h}+\beta_{k}\right)\right)}{\Gamma(\alpha+1)}\left[1+\frac{|b|}{|a+b|}\right]+\frac{|c|}{|a+b|} \leqslant T<1 .
$$

Hence $U_{k}$ tends to zero as $k$ tends to infinity. Since the family $\left\{U_{k}\right\}$ is the Arzelà-Ascoli family thus for every subsequence $\left\{u_{k j}\right\}$ of $\left\{U_{k}\right\}$ there exists a subsequence $\left\{u_{k j}\right\}$ uniformly convergent and the limit needs to be a solution of the problem (1)-(2). Thus, the sequence $\left\{U_{k}\right\}$ tends uniformly to the unique solution of the problem (1)-(2).

\section{Stability results}

In this section, we investigate the Ulam-Hyers stability and generalized Ulam-Hyers stability for the problem (1)-(2).

Definition 3.1 ([27]). The Eq. (1) is Ulam-Hyers stable if there exists a real number $\Omega>0$ such that for each $\epsilon>0$ and for each solution $v \in C^{1}(J, J)$ of the inequality

$$
\left|D^{\alpha} v(x)-f(x)-\int_{0}^{x} h(x, s) v(v(s)) d s-\int_{0}^{T} k(x, s) v(v(s)) d s\right| \leqslant \epsilon, \quad x \in J,
$$

there exists a solution $u \in C^{1}(J, J)$ of Eq. (1) with

$$
|v(x)-u(x)| \leqslant \Omega \epsilon .
$$

Definition 3.2 ([27]). The Eq. (1) is generalized Ulam-Hyers stable if there exists $\Theta \in$ $C\left(\mathbb{R}^{+}, \mathbb{R}^{+}\right), \quad \Theta(0)=0$ such that for each $\epsilon>0$ and for each solution $v \in C^{1}(J, J)$ of the inequality

$$
\left|D^{\alpha} v(x)-f(x)-\int_{0}^{x} h(x, s) v(v(s)) d s-\int_{0}^{T} k(x, s) v(v(s)) d s\right| \leqslant \epsilon, \quad x \in J,
$$

there exists a solution $u \in C^{1}(J, J)$ of $E q$. (1) with

$$
|v(x)-u(x)| \leqslant \Theta(\epsilon) .
$$


Theorem 3.1. If the assumptions of the Theorem 2.1 are satisfied, then the problem (1)-(2) is Ulam-Hyers stable.

Proof. Let $\epsilon>0$ and let function $v \in C^{1}(J, J)$ which satisfies the inequality

$$
D^{\alpha} v(x)-f(x)-\int_{0}^{x} h(x, s) v(v(s)) d s-\int_{0}^{T} k(x, s) v(v(s)) d s \leqslant \epsilon,
$$

and let $u \in C(J, J)$ be the unique solution of the following problem

$$
\begin{aligned}
& D^{\alpha} u(x)=f(x)+\int_{0}^{x} h(x, s) u(u(s)) d s+\int_{0}^{T} k(x, s) u(u(s)) d s, \\
& u(0)=v(0), \quad u(T)=v(T) .
\end{aligned}
$$

from Lemma 2.1, we obtain

$$
\begin{aligned}
u(x) & =\int_{0}^{x} \frac{(x-t)^{\alpha-1}}{\Gamma(\alpha)}\left(f(t)+\int_{0}^{t} h(t, s) u(u(s)) d s+\int_{0}^{T} k(t, s) u(u(s)) d s\right) d t- \\
& -\frac{1}{a+b}\left[\int_{0}^{T} \frac{b(T-t)^{\alpha-1}}{\Gamma(\alpha)}\left(f(t)+\int_{0}^{t} h(t, s) u(u(s)) d s+\int_{0}^{T} k(t, s) u(u(s)) d s\right) d t-c\right]= \\
& =\Delta_{u}+\int_{0}^{x} \frac{(x-t)^{\alpha-1}}{\Gamma(\alpha)}\left(f(t)+\int_{0}^{t} h(t, s) u(u(s)) d s+\int_{0}^{T} k(t, s) u(u(s)) d s\right) d t,
\end{aligned}
$$

where

$$
\Delta_{u}=\frac{1}{a+b}\left[c-\int_{0}^{T} \frac{b(T-t)^{\alpha-1}}{\Gamma(\alpha)}\left(f(t)+\int_{0}^{t} h(t, s) u(u(s)) d s+\int_{0}^{T} k(t, s) u(u(s)) d s\right) d t\right] .
$$

Let

$$
\Delta_{v}=\frac{1}{a+b}\left[c-\int_{0}^{T} \frac{b(T-t)^{\alpha-1}}{\Gamma(\alpha)}\left(f(t)+\int_{0}^{t} h(t, s) v(v(s)) d s+\int_{0}^{T} k(t, s) v(v(s)) d s\right) d t\right] .
$$

On the other hand, if $u(0)=v(0), u(T)=v(T)$, then $\Delta_{u}=\Delta_{v}$ and

$$
u(x)=\Delta_{v}+\int_{0}^{x} \frac{(x-t)^{\alpha-1}}{\Gamma(\alpha)}\left(f(t)+\int_{0}^{t} h(t, s) u(u(s)) d s+\int_{0}^{T} k(t, s) u(u(s)) d s\right) d t .
$$

From inequality (11) we have

$$
-\epsilon \leqslant D^{\alpha} v(x)-f(x)-\int_{0}^{x} h(x, s) v(v(s)) d s-\int_{0}^{T} k(x, s) v(v(s)) d s \leqslant \epsilon .
$$

If we integrate each term of the above inequality and appling the boundary conditions, then we have

$$
\left|v(x)-\Delta_{v}-\int_{0}^{x} \frac{(x-t)^{\alpha-1}}{\Gamma(\alpha)}\left(f(t)+\int_{0}^{t} h(t, s) v(v(s)) d s+\int_{0}^{T} k(t, s) v(v(s)) d s\right) d t\right| \leqslant \frac{\epsilon T^{\alpha}}{\Gamma(\alpha+1)} .
$$

For any $x \in J$, we have

$$
\begin{aligned}
& |v(x)-u(x)| \leqslant \\
\leqslant & \left|v(x)-\Delta_{v}-\int_{0}^{x} \frac{(x-t)^{\alpha-1}}{\Gamma(\alpha)}\left(f(t)+\int_{0}^{t} h(t, s) v(v(s)) d s+\int_{0}^{T} k(t, s) v(v(s)) d s\right) d t\right|+
\end{aligned}
$$




$$
\begin{aligned}
& +\int_{0}^{x} \frac{(x-t)^{\alpha-1}}{\Gamma(\alpha)}\left(\int_{0}^{t} h(t, s)|v(v(s))-u(u(s))| d s+\int_{0}^{T} k(t, s)|v(v(s))-u(u(s))| d s\right) d t \leqslant \\
& \leqslant \frac{\epsilon T^{\alpha}}{\Gamma(\alpha+1)}+\int_{0}^{x} \frac{(x-t)^{\alpha-1}}{\Gamma(\alpha)}\left(\beta_{h} \int_{0}^{t}[|v(v(s))-v(u(s))|+|v(u(s))-u(u(s))|] d s+\right. \\
& \left.+\beta_{k} \int_{0}^{T}[|v(v(s))-v(u(s))|+|v(u(s))-u(u(s))|] d s\right) d t \leqslant \\
& \leqslant \frac{\epsilon T^{\alpha}}{\Gamma(\alpha+1)}+\frac{\beta_{h}}{\Gamma(\alpha)} \int_{0}^{x} \int_{0}^{t}(x-t)^{\alpha-1}(M+1)|v(s)-u(s)| d s d t+ \\
& +\frac{\beta_{k}}{\Gamma(\alpha)} \int_{0}^{x} \int_{0}^{T}(x-t)^{\alpha-1}(M+1)|v(s)-u(s)| d s d t \leqslant \\
& \leqslant \frac{\epsilon T^{\alpha}}{\Gamma(\alpha+1)}+\frac{T^{\alpha}\left(\beta_{h}+\beta_{k}\right)(M+1)}{\Gamma(\alpha+1)} \int_{0}^{x}|v(s)-u(s)| d s .
\end{aligned}
$$

Using Gronwall's inequality, we get

$$
|v(x)-u(x)| \leqslant \frac{\epsilon T^{\alpha}}{\Gamma(\alpha+1)}\left[1+\frac{\xi T^{\alpha}\left(\beta_{h}+\beta_{k}\right)(M+1)}{\Gamma(\alpha+1)}\right]:=\Omega \epsilon,
$$

where $\xi=\xi(\alpha)$ a constant, which completes the proof.

Moreover, if we set $\Theta(\epsilon)=\Omega \epsilon, \Theta(0)=0$, then boundary value problem (1)-(2) is generalized Ulam-Hyers stable.

\section{An example}

We consider the nonlinear iterative fractional integro-differential equation (1)-(2) with

$$
\alpha=0.5, \quad T=0.5, \quad L=0.2, \quad M=0.4, \quad \beta_{h}=\beta_{k}=0.5, \quad a=b=1, \quad \text { and } \quad c=0 .
$$

New, we have

$$
\begin{aligned}
\frac{T^{\alpha}\left(L+T^{3}\left(\beta_{h}+\beta_{k}\right)\right)}{\Gamma(\alpha+1)}\left(1+\frac{|b|}{|a+b|}\right)+\frac{|c|}{|a+b|} & =\frac{0.5^{0.5}\left(0.2+0.5^{3}(0.5+0.5)\right)}{\Gamma(0.5+1)}\left(1+\frac{1}{2}\right)+0 \\
& =\frac{0.2298098}{\Gamma(1.5)}(1.5) \\
& =\frac{0.3447145}{0.886227} \\
& =0.38897 \\
& <0.5=T .
\end{aligned}
$$

Also,

$$
\begin{aligned}
\frac{T^{\alpha+1}(M+1)\left(\beta_{h}+\beta_{k}\right)}{\Gamma(\alpha+1)}\left(1+\frac{|b|}{|a+b|}\right) & =\frac{0.5^{0.5+1}(0.4+1)(0.5+0.5)}{\Gamma(0.5+1)}\left(1+\frac{1}{2}\right) \\
& =\frac{0.494975}{0.886227}(1.5) \\
& =0.8378 \\
& <1 .
\end{aligned}
$$

Since all the hypotheses of Theorem 2.1 are fulfilled, then there exists a unique solution of the given equation. 


\section{Conclusion}

The main purpose of this paper was to present new existence and uniqueness results as well as the Ulam-Hyers stability and generalized Ulam-Hyers stability results of the solution for Caputo fractional iterative Volterra-Fredholm integro-differential. The techniques used to prove our results are a variety of tools such as the Gronwall-Bellman's inequality, some properties of fractional calculus and the Banach contraction fixed point theorem. Moreover, the results of references $[15,16,28]$ appear as a special case of our results.

The author would like to thank the referees for their valuable suggestions and comments that improved this paper.

\section{References}

[1] B.Ahmad, S.Sivasundaram, Some existence results for fractional integro-differential equations with nonlinear conditions, Communications Appl. Anal., 12(2008), 107-112.

[2] M.Ali, W.Ma, New exact solutions of nonlinear (3+1)-dimensional Boiti-Leon-MannaPempinelli equation, Advances in Mathematical Physics, 2019(2019), 1-8.

[3] M.Ali, A.Hadhoud, H.Srivastava, Solution of fractional Volterra-Fredholm integrodifferential equations under mixed boundary conditions by using the HOBW method, Advances in Mathematical Physics, 2019(2019), no. 1, 1-14. DOI: 10.1155/2019/9801638

[4] M.Bani Issa, A.Hamoud, K.Ghadle, Numerical solutions of fuzzy integro-differential equations of the second kind, Journal of Mathematics and Computer Science, 23(2021), 67-74. DOI: $10.22436 /$ jmcs.023.01.07

[5] S.Cheng, S.Talwong, V.Laohakosol, Exact solution of iterative functional differential equation, Computing, 76(2006), 67-76. DOI: 10.1007/s00607-005-0127-1

[6] L.Dawood, A.Sharif, A.Hamoud, Solving higher-order integro-differential equations by VIM and MHPM, International Journal of Applied Mathematics, 33(2020), 253-264. DOI: 10.12732 /ijam.v33i2.5

[7] A.Hamoud, K.Ghadle, The approximate solutions of fractional Volterra-Fredholm integrodifferential equations by using analytical techniques, Probl. Anal. Issues Anal., 7(25)(2018), no. $1,41-58$. DOI: $10.15393 /$ j3.art.2018.4350

[8] A.Hamoud, K.Ghadle, Usage of the homotopy analysis method for solving fractional Volterra-Fredholm integro-differential equation of the second kind, Tamkang J. Math., 49(2018), no. 4, 301-315. DOI: 10.5556/j.tkjm.49.2018.2718

[9] A.Hamoud, K.Hussain, K.Ghadle, The reliable modified Laplace Adomian decomposition method to solve fractional Volterra-Fredholm integro differential equations, Dynamics of Continuous, Discrete and Impulsive Systems, Series B: Applications \& Algorithms, 26(2019), 171-184.

[10] A.Hamoud, K.Ghadle, Existence and uniqueness of the solution for Volterra-Fredholm integro-differential equations, Journal of Siberian Federal University. Mathematics \& Physics, 11(2018), no. 6, 692-701. DOI: 10.17516/1997-1397-2018-11-6-692-701

[11] A.Hamoud, K.Ghadle, Some new existence, uniqueness and convergence results for fractional Volterra-Fredholm integro-differential equations, J. Appl. Comput. Mech., 5(2019), no. 1, 58-69. DOI: $10.22055 /$ jacm.2018.25397.1259 
Ahmed A. Hamoud Uniqueness and Stability Results for Caputo Fractional Volterra-Fredholm ...

[12] A.Hamoud, Existence and uniqueness of solutions for fractional neutral Volterra-Fredholm integro-differential equations, Advances in the Theory of Nonlinear Analysis and its Application, 4(2020), no.4, 321-331. DOI: 10.31197/atnaa.799854

[13] J.H.He, Some applications of nonlinear fractional differential equations and their approximations, Bull. Sci. Technol. Soc., 15(1999), 86-90.

[14] R.Ibrahim, S.Momani, On the existence and uniqueness of solutions of a class of fractional differential equations, Journal of Mathematical Analysis and Applications, 334(2007), 1-10. DOI: $10.1016 /$ j.jmaa.2006.12.036

[15] R.Ibrahim, Existence of iterative Cauchy fractional differential equations, Int. J. Math. Sci., 7(2013), 379-384.

[16] S.Kendre, V.Kharat, R.Narute, On existence of solution for iterative integro-differential equations, Nonlinear Anal. Differ. Equ., 3(2015), 123-131.

[17] A.Kilbas, H.Srivastava, J.Trujillo, Theory and Applications of Fractional Differential Equations, North-Holland Math. Stud., Elsevier, Amsterdam, Vol. 204, 2006.

[18] V.Lakshmikantham, M.Rao, Theory of Integro-Differential Equations, Gordon \& Breach, London, 1995.

[19] M.Lauran, Existence results for some differential equations with deviating argument, Filomat, 25(2011), 21-31. DOI: 10.2298/FIL1102021L

[20] W.Li, S.Cheng, A picard theorem for iterative differential equations, Demonstratio Math., 42(2009), 371-380.

[21] S.Muthaiah, M Murugesan, N.Thangaraj, Existence of solutions for nonlocal boundary value problem of Hadamard fractional differential equations, Adv. Theory Nonlinear Anal. Appl., 3(2019), 162-173. DOI: 10.31197/atnaa.579701

[22] M.Matar, Controllability of fractional semilinear mixed Volterra-Fredholm integro differential equations with nonlocal conditions, Int. J. Math. Anal., 4(2010), 1105-1116.

[23] S.Momani, A.Jameel, S.Al-Azawi, Local and global uniqueness theorems on fractional integro-differential equations via Bihari's and Gronwall's inequalities, Soochow J. Math., 33(2007), 619-627.

[24] K.Miller, B.Ross, An Introduction to the Fractional Calculus and Differential Equations, John Wiley, New York, 1993.

[25] R.Panda, M.Dash, Fractional generalized splines and signal processing, Signal Process, 86(2006), 2340-2350.

[26] S.Samko, A.Kilbas, O.Marichev, Fractional Integrals and Derivatives, Theory and Applications, Gordon and Breach, Yverdon, 1993.

[27] R.Shah, A.Zada, A fixed point approach to the stability of a nonlinear Volterra integrodifferential equations with delay, Hacet. J. Math. Stat., 47(2018), 615-623.

[28] S.Unhaley, S.Kendre, On existence and uniqueness results for iterative fractional integrodifferential equation with deviating arguments, Applied Mathematics E-Notes, 19(2019), $116-127$. 
[29] J.Wu, Y.Liu, Existence and uniqueness of solutions for the fractional integro-differential equations in Banach spaces, Electron. J. Differential Equations, 2009(2009), 1-8.

\title{
Результаты единственности и устойчивости для Капуто дробных интегро-дифференциальных уравнений Вольтерра-Фредгольма
}

\author{
Ахмед А. Хамуд \\ Кафедра математики \\ Университет Таиз \\ Таиз, Йемен
}

\begin{abstract}
Аннотация. В этой статье мы установили некоторые новые результаты, касающиеся единственности и устойчивости Улама решений итерационных нелинейных интегро-дифференциальных уравнений Вольтерра-Фредгольма с граничными условиями. Дробные производные рассматриваются в смысле Капуто. Эти новые результаты получены путем применения неравенства ГронуоллаБеллмана и теоремы Банаха о сжатии неподвижной точки. Включен наглядный пример, чтобы продемонстрировать эффективность и надежность результатов.
\end{abstract}

Ключевые слова: интегро-дифференциальное уравнение Вольтерра-Фредгольма, смысл Капуто, неравенство Гронуолла-Беллмана, теорема Банаха о сжатии неподвижной точки. 